\title{
The Starburst-AGN connection: quenching the fire and feeding the monster
}

\author{
Jorge Melnick ${ }^{1,2}$, Eduardo Telles ${ }^{2}$, Roberto De Propris ${ }^{3}$, and Zhang-Hu Chu ${ }^{4}$ \\ ${ }^{1}$ European Southern Observatory, Av. Alonso de Cordova 3107, Santiago, Chile \\ e-mail: jmelnick@eso.org \\ 2 Observatorio Nacional, Rua José Cristino 77, 20921-400 Rio de Janeiro, Brasil \\ 3 Finnish Centre for Astronomy with ESO, University of Turku, Väisäläntie 20, 21500 Piikkiö, Finland \\ 4 Department of Astronomy, Nanjing University, Nanjing 210093, PR China
}

Received 26 March 2015 / Accepted 3 July 2015

\begin{abstract}
The merger of two spiral galaxies is believed to be one of the main channels for the production of elliptical and early-type galaxies. In the process, the system becomes an (ultra) luminous infrared galaxy, or (U)LIRG, that morphs to a quasar, to a $\mathrm{K}+\mathrm{A}$ galaxy, and finally to an early-type galaxy. The time scales for this metamorphosis are only loosely constrained by observations. In particular, the $\mathrm{K}+\mathrm{A}$ phase should follow immediately after the quasi stellar object (QSO) phase during which the dust and gas remaining from the (U)LIRG phase are expelled by the active galactic nucleus (AGN). An intermediate class of QSOs with $\mathrm{K}+\mathrm{A}$ spectral signatures, the post-starburst QSOs (PSQ), may represent the transitional phase between QSOs and K+As. We have compiled a sample of 72 bona fide $z<0.5$ PSQ from the SDSS DR7 QSO catalogue. We find the intermediate age populations in this sample to be on average significantly weaker and metal poorer than their putative descendants, the $\mathrm{K}+\mathrm{A}$ galaxies. The typical spectral energy distribution of PSQ is well fitted by three components: starlight; an obscured power-law; and a hot dust component required to reproduce the mid-IR fluxes. From the slope and bolometric luminosity of the power-law component we estimate typical masses and accretion rates of the AGN, but we find little evidence of powerful radio-loud or strong X-ray emitters in our sample. This may indicate that the power-law component originates in a nuclear starburst rather than in an AGN, as expected if the bulk of their young stars are still being formed, or that the AGN is still heavily enshrouded in dust and gas. We find that both alternatives are problematic and that more and better optical, X-ray, and mm-wave observations are needed to elucidate the evolutionary history of PSQ.
\end{abstract}

Key words. quasars: absorption lines - quasars: general - galaxies: interactions - galaxies: nuclei - galaxies: starburst galaxies: star formation

\section{Introduction}

The possibility that at least some elliptical galaxies could be the end result of the merger of two spirals of similar masses was first proposed by Toomre \& Toomre (1972; see also Toomre 1977) and has since been explored in numerous investigations (see e.g. Hopkins et al. 2008a, for a recent review). While intriguing, the idea of the metamorphosis of spirals into ellipticals has been questioned on dynamical and photometric grounds. Nevertheless, one by one these objections have been resolved by increasingly sophisticated observations and numerical modelling.

The main difficulty lies in suppressing star formation in the merger of two gas-rich systems to return a quiescent remnant. This process (quenching) may be aided by feedback processes, and especially by the activation of an active nucleus (expected if mergers drive gas and dust to the centre by tidal torques e.g. Mihos \& Hernquist 1996). In the detailed simulations by Hopkins et al. (2008a,b) the merger of two gas-rich systems produces an active galactic nucleus (AGN) that eventually stops or prevents further star formation. The resulting remnant goes through a brief AGN phase and is then identifiable as a poststarburst $(\mathrm{K}+\mathrm{A})$ galaxy as the initial star formation episode fades away. A recent investigation has revealed that a median $50 \%$ of the stellar mass in a sample of $808 \mathrm{~K}+\mathrm{A}$ galaxies has intermediate ages and high metallicities (Melnick \& De Propris 2014), consistent in fact with the metallicities of elliptical galaxies of similar masses. Thus, the present day stellar populations of at least some elliptical galaxies appear to have been formed in a colosal burst of star formation induced by the merger of two gas-rich galaxies of similar masses (the preferred model for the origin of K+A galaxies; Snyder et al. 2011).

The signatures of this process may be difficult to identify. There are several lines of evidence now favouring a secular scenario for AGN evolution (e.g. Jahnke \& Macció 2011). X-ray selected AGN hosts in deep HST imaging are not observed to be more disturbed than a similar sample of inactive galaxies at the same redshifts (e.g. Cisternas et al. 2011; Kocevski et al. 2012; Villforth et al. 2014, and references therein), while both De Propris et al. (2014) and Scott \& Kaviraj (2014) do not find evidence for a strong enhancement of the merging fraction in closely interacting pairs, despite the presence of tidal signatures. Similarly, Teng et al. (2012) searched for AGN activity in companion galaxies to quasi stellar object (QSO) hosts and detected just one AGN in their sample of 12 galaxies.

It is of course possible that early QSOs are highly obscured: Koss et al. (2011) shows that hard X-ray selected AGNs, which are often Compton thick, are hosted by highly disturbed galaxies, while Urrutia et al. (2008) find that a large fraction of Type II (red, dust obscured) QSOs lie in morphologically disturbed systems. Canalizo \& Stockton (2013) argue that QSOs with strong absorption lines have evidence of tidal interaction, but this is 

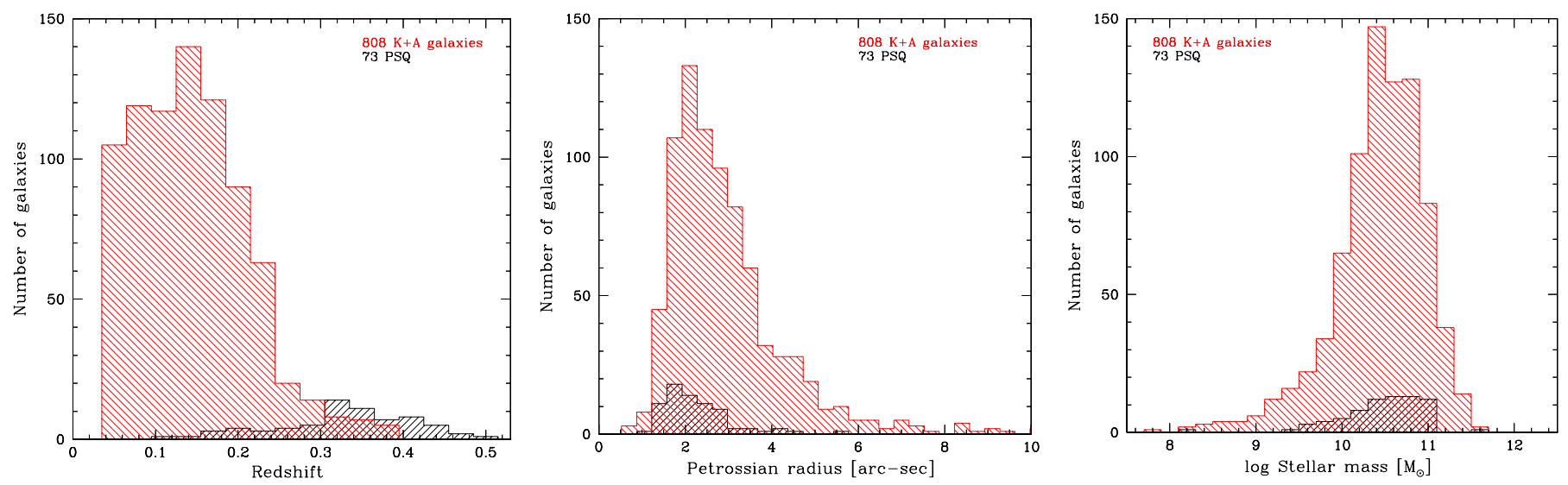

Fig. 1. Statistical properties of the PSQ sample (in black) compared to those of their putative descendants the K+A galaxies (in red).

only present at low surface brightness levels, which argues for gas-poor, low mass, or even old remnants, that may or may not be connected with the observed present-day activity.

If we accept the QSO-merger model, we expect that at least some young QSOs may be associated with a post-starburst signature as they have just suppressed star formation and are emerging from their dust-enshrouded phase. In a previous paper (De Propris \& Melnick 2014) we have shown that no $\mathrm{K}+\mathrm{A}$ galaxy at any age hosts an AGN, although there is clear evidence of feedback (see also Wong et al. 2012 for a similar conclusion). Therefore, suppression of star formation and QSO activity must rapidly follow each other (see e.g. Teng et al. 2012). Thus, the particular class of quasars known as post-starburst quasars (PSQ; see Cales et al. 2013, for a recent review), would be the immediate ancestors of $\mathrm{K}+\mathrm{A}$ galaxies and therefore the descendants of recent mergers.

Observationally, PSQ are characterised by strong emission lines and strong absorption of the high Balmer lines. Cales et al. (2013) concluded that PSQ with early-type hosts are likely the result of major mergers, so this class of objects may offer a way to witness the final stages of the metamorphosis of spirals into early-type galaxies. In this paper we present a systemic study of the properties of a sample of 72 PSQ from the SDSS selected from the DR7 Catalog of Quasars (Schneider et al. 2010), paralleling our study of $\mathrm{K}+\mathrm{A}$ galaxies. We use the SDSS spectra to derive the stellar populations, which are then used to predict the broad-band spectral energy distributions (SEDs) from the far ultraviolet (FUV) to the mid infrared (mid-IR). Throughout this work we adopt the following cosmological parameters: $\Omega_{\mathrm{M}}=$ $0.3, \Omega_{\Lambda}=0.7$, and $H_{0}=70 \mathrm{~km} \mathrm{~s}^{-1} \mathrm{Mpc}^{-1}$.

\section{Data and models}

The DR7 Quasar Catalog (DR7Q) contains data for 105,783 SDSS quasars selected as described by Schneider et al. (2010). We downloaded the SDSS spectra of the 8492 objects in the DR7Q with $z \leq 0.5$, which we searched for signatures of post-starburst stellar populations using a slightly more relaxed criterion than that used by Goto (2006): we looked for objects with (rest frame) equivalent withs of $\mathrm{H} \delta$ greater than $E W(H \delta)>3 \AA$ in absorption. We found that about $1.5 \%$ of the objects (125 out of 8492) satisfied this criterion.

Our PSQ fraction is significantly lower than the fraction of $4.2 \%$ PSQs found by Goto (2006) in a volume limited sample of SDSS galaxies. This is not surprising given that Goto gives the fraction of all AGN that have strong $\mathrm{H} \delta$ in absorption, while our sample is restricted to objects classified as QSOs, that is, only to AGN with broad emission lines. In fact, the three examples shown by Goto (2006) are classified as (Seyfert 2) galaxies in the SDSS and therefore are not included in the DR7Q.

We visually inspected the spectra for spurious detections, which led to a further reduction of the sample to 83 objects; while some were clearly not PSQs, the signal-to-noise ratios $(\mathrm{S} / \mathrm{N})$ of others was not sufficient to ascertain the presence of strong $\mathrm{H} \delta$ in absorption. Finally we ran the STARLIGHT code (Cid Fernandes et al. 2005) to model the stellar populations of these 83 objects using exactly the same prescriptions of Melnick \& De Propris (2014) for the K+A galaxies: BC03 (Bruzual \& Charlot 2003) stellar library for 25 ages and 5 metallicities. From the STARLIGHT fits we found that the SDSS spectra of 11 out of our 83 bona fide PSQs were of insufficient quality to derive reliable stellar populations, either because the $\mathrm{S} / \mathrm{N}$ was too low ( $S / N<2$ at $4100 \AA$ ), or due to significant gaps at some critical wavelengths.

The essential statistical properties of our final sample of 72 PSQ are presented in Fig. 1 in comparison the properties of the K+A galaxies from Melnick \& De Propris (2013). The comparison is relevant because the main purpose of our investigation is to establish whether PSQ can be the progenitors of $\mathrm{K}+\mathrm{As}$.

Figure 1 shows that - as QSOs - PSQ are rare in the local Universe, even compared to $\mathrm{K}+\mathrm{A}$ galaxies. On the other hand, the stellar masses of PSQ appear to be comparable to, those of $\mathrm{K}+$ As. More surprising is that the Petrossian radii of the two samples are comparable given the differences in redshift. While this is probably due to the relatively low resolution of the SDSS images, it confirms that, as discussed by Melnick \& De Propris (2013), the photometry and spectroscopy of our PSQ sample are not significantly affected by systematic aperture matching effects.

The star formation histories for our 72 bona fide PSQs were combined to build the SEDs of the stellar components from the FUV to the mid-IR using the BC03 stellar library to convert masses and ages to photometric fluxes, which we compared to the photometric observations. We emphasise that we do not use the spectral fluxes to construct the SEDs, but the stellar populations derived from the spectra. This allows us to effectively extend the spectral coverage to the FUV and mid-IR.

The essential characteristics of the broad-band photometric dataset are: optical (ugriz) from the SDSS (Abazajian et al. 2009); Vacuum UV data are from the GALEX imaging databases (Morrissey et al. 2007); Near-IR data in JHK come from 2MASS (Skrutskie et al. 2006); and Mid-infrared data from 

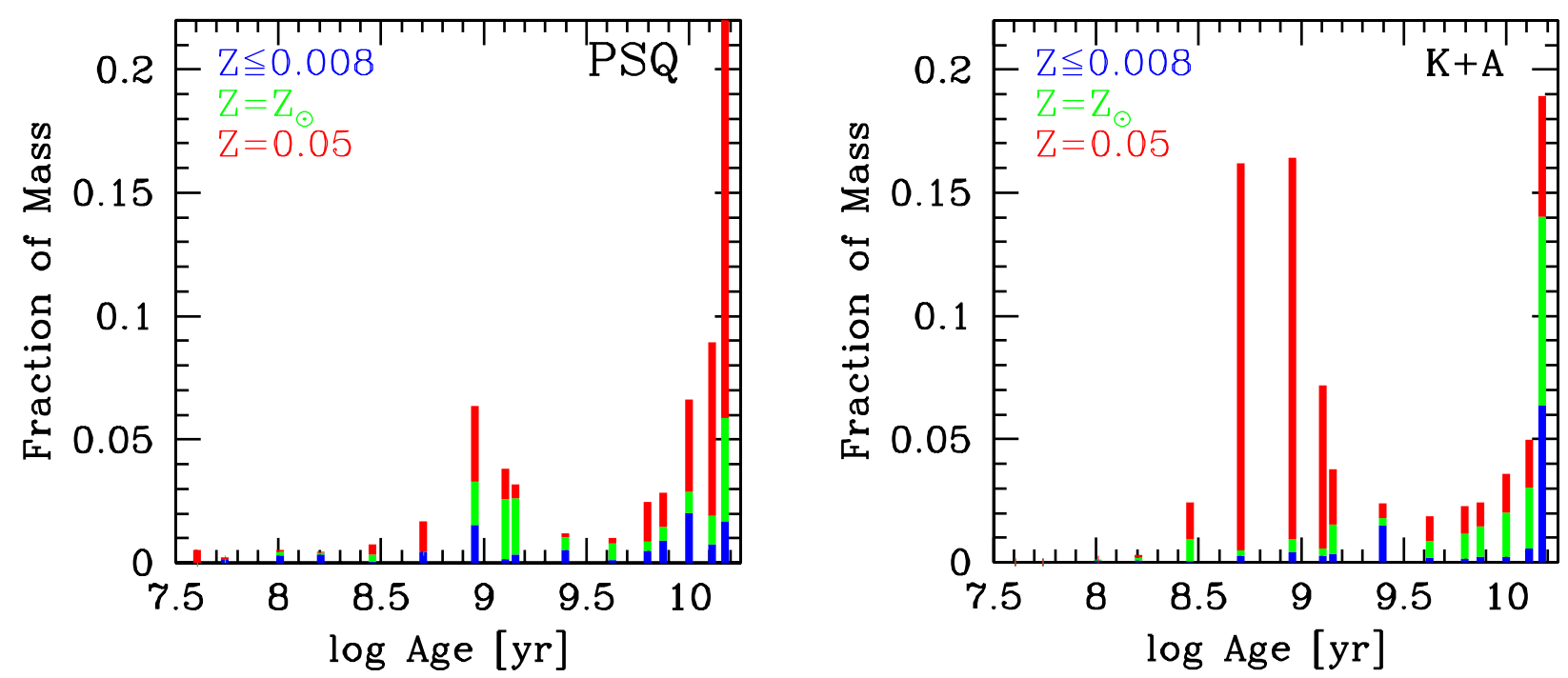

Fig. 2. Comparison between the stellar populations of PSQ on the left and of K+A galaxies on the right for three broad metallicity bins.

the WISE (Wright et al. 2009) datasets. The photometry and the input SDSS spectra were corrected for Galactic foreground extinction using the values of given in the DR7Q (Schneider et al. 2010) using the standard Galactic reddening law (Cardelli et al. 1989; henceforth CCM).

The synthetic SEDs are calculated as

$F(\lambda)=k \sum_{i=1}^{25} \sum_{j=1}^{5} S_{i j} M_{i j}(\lambda)$

where $M_{i j}$ are the $\mathrm{BC} 03$ models for the $i=1, \ldots, 25$ age bins and $j=1, \ldots, 5$ metallicity bins, and $S_{i j}$ are the mass fractions returned by STARLIGHT for the relevant age and metallicity bins. The photometric zero-point $k$ is independent of wavelength.

\section{Results}

\subsection{Stellar populations}

Figure 2 presents in the left panel the average stellar populations of the full sample of 72 PSQ analysed with STARLIGHT. These plots give fractions of the total stellar mass as a function of age for three broad metallicity bins (metal poor; solar; oversolar) chosen to make the plot less cluttered. For comparison, the right panel presents the mean stellar populations of our sample of $808 \mathrm{~K}+\mathrm{A}$ galaxies from Melnick \& De Propris (2014). Two remarkable differences are immediately obvious: (1) the average fraction of mass in intermediate age stars is about $15 \%$ in PSQ compared to $\sim 45 \%$ for $\mathrm{K}+\mathrm{A}$ galaxies; and (2), while in $\mathrm{K}+\mathrm{As}$ the vast majority of the intermediate-age stars are metal rich, in PSQ this is the case for only a small fraction of the stars.

It is notoriously difficult to estimate formal statistical errors for the stellar populations derived from population synthesis modelling (see e.g. Melnick \& De Propris 2014, for a discussion in the case of $\mathrm{K}+\mathrm{A}$ galaxies and STARLIGHT). Systematics dominate over formal fitting errors, in particular the well known degeneracies between age and metallicity. In the case of PSQ one should be particularly concerned by the effect of a featureless power-law continuum from the AGN.

STARLIGHT offers various possibilities to include a powerlaw continuum, and in particular to deal with different extinctions for different populations. We ran many experiments to establish whether a combination of power-law and extinction could affect the resulting stellar populations. We found that, while including these combinations may change the detailed distribution of ages and metallicities, the total fractions of intermediate age stars remained substantially unchanged.

We obtained very consistent results by either including a power-law component of slope $\alpha=-1.5\left(f_{\lambda} \propto(\lambda / 0.55 \mu)^{-1.5}\right)$ reddened by $A_{V}=1.3 \mathrm{mag}$, as determined from our SED fitting discussed in the next section, or by including an un-reddened power-law of the same slope and allowing STARLIGHT to choose the best fitting extinction for each object. The former is shown in Fig. 2, and is the one we used to compute the total stellar masses, but the differences between the two methods are surprisingly small.

From these and the other experiments we ran, we are confident that the differences between the stellar populations of PSQ and $\mathrm{K}+$ As shown in Fig. 2 are not due to our choice of how to model the power-law continuum.

Another systematic effect is contamination of the Balmer stellar lines by nebular emission, which could result in lower fractions of intermediate-age stars. Visual inspection of the spectra shows this effect to be present in some of our objects, but even at $\mathrm{H} \delta$ (we mask $\mathrm{H} \alpha, \beta, \gamma$ ) the contamination is very small. In order to quantify this effect we subtracted the population synthesis model from the original spectra to generate a pure nebular spectrum. We then subtracted the smoothed nebular spectrum from the observed spectrum to generate an emission-line free spectrum, which we fitted again with STARLIGHT.

This procedure inevitably adds noise to our already rather noisy SDSS spectra. We have tested the procedure with rather discouraging results: we get no net increase in the fraction of intermediate-mass stars and the metallicities do not change significantly. Masking out $\mathrm{H} \delta$ in the original spectra gave similar negative results. We also tested stacking the spectra of all the objects that allows us to remove the emission lines from the stacked spectrum without adding noise. The change in the stellar populations with and without removing the emission lines was negligible and $\mathrm{EW}(\mathrm{H} \delta)$ changed by less than $10 \%$.

We performed a number of other tests to evaluate the systematical errors in our results: changing spectral range to eliminate the noisiest spectral regions; changing the reddening law; and masking various spectral features. While the stellar populations change in detail, the overall fractions and metallicities 


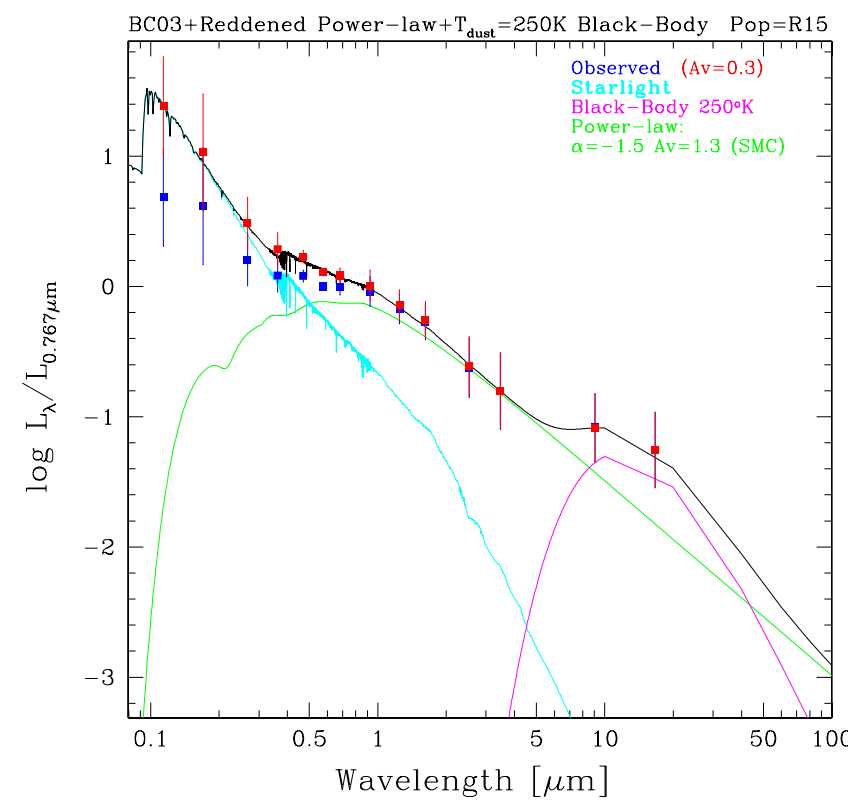

Fig. 3. Best fit model SED for the average of the $72 \mathrm{~K}+\mathrm{A}$ galaxies in our sample compared to the average observed broad-band fluxes corrected for extinction as described in the text and normalised to the SDSS $i^{\prime}$-band $\left(F_{0.767 \mu \mathrm{m}}\right)$. The blue symbols show the observed photometric data points and the red symbols show the observations corrected for extinction using the average visual extinction shown in parenthesis in the figure legend and the GD1 extinction law. The three components of the fit are colour-coded as follows: cyan shows the contribution of the stars computed from the STARLIGHT population-synthesis fits to the SDSS spectra; green shows the obscured power-law; and the magenta represents the $T_{\text {dust }}=250 \mathrm{~K}$ hot dust component, which is required by the mid-IR data. The sum of these three components is shown in black. The error bars are not observational errors but correspond to the $1 \sigma$ dispersions of the normalised fluxes for all the objects included in each band, the number of which varies from 45 in the FUV band to 72 for the SDSS bands. The reddened power-law component is the one included in the population synthesis models, thus the label Pop = R15 in the figure legend.

of the intermediate-age component never changed significantly and in particular never increased. We are confident, therefore, that the discrepancy between PSQ and $\mathrm{K}+\mathrm{As}$ is not due to errors in the fitting procedure.

\subsection{Synthetic SEDs}

Figure 3 compares the average synthetic SED computed using Eq. (1) for the mean of the STARLIGHT stellar populations (shown in Fig. 2) to the observations. In order to remove the intrinsic variance in luminosity we normalised the fluxes to the SDSS $i^{\prime}$-band $\left(F_{0.767 \mu \mathrm{m}}\right)$. The observed fluxes show a clear excess above $\lambda \sim 0.5 \mu$, which is very different from the case of $\mathrm{K}+\mathrm{As}$ where the excess is only observed in the mid-IR bands at $\lambda>5 \mu$. In order to fit the PSQ, therefore, in addition to a hot-dust component parametrised as a black-body of temperature $T_{\text {dust }}$ as we did for $\mathrm{K}+\mathrm{A}$ galaxies, we added a reddened power-law component as expected for AGN.

We emphasise that the error bars plotted in the figure are not observational photometric errors, but the root-mean square scatter of the normalised fluxes about the mean of the sample. The mean observational errors range from 0.26 mag at $22 \mu$ and $0.23 \mathrm{mag}$ in FUV to $<0.03 \mathrm{mag}$ in the $\operatorname{SDSS} g^{\prime}$ to $z^{\prime}$ bands. Thus, while PSQ form a remarkably homogeneous class of objects at optical wavelengths, this is not the case in the UV and IR bands. This is different from $\mathrm{K}+\mathrm{A}$ galaxies that have remarkably similar SEDs in the optical and in the IR up to $4.5 \mu$ (Melnick \& De Propris 2014).

We thus need six free parameters to fit the observed SEDs: $T_{\text {dust }} ; \alpha_{\mathrm{UV}}$ - the slope of the power-law component; and $A_{V}^{\mathrm{PL}}-$ the visual extinction of the power-law, plus the photometric zeropoints of the three components: stellar populations; obscured power-law; and hot-dust. An additional free parameter is the reddening law for the stellar and power-law components, which is very relevant for the UV bands.

In principle the extinction is a free parameter only for the power-law component. In the case of $\mathrm{K}+\mathrm{A}$ galaxies we corrected the photometric observations using the extinction values from the STARLIGHT fits. However, this is not possible, or at least is not straightforward, in the case of PSQ because the power-law component contributes a significant fraction of the continuum flux in the SDSS spectral band. We verified that this does not change the stellar populations significantly, but it certainly affects the resulting extinction, which in turn affect the SED fits and thus the optical power-law continuum. We tested an iterative procedure, which is very time consuming and does not easily converge.

We therefore followed a different procedure to estimate the extinction corrections for the stellar component, $A_{V}^{*}$. First we fixed the photometric zero points of the power-law and hot-dust components by forcing each to fit the WISE $4.5 \mu$ and $12 \mu$ bands respectively; the power-law completely dominates the $4.5 \mu$ flux and the Black-Body completely dominates the $12 \mu$ emission, with negligible extinction corrections. For the stellar component we fixed the zero point in the SDSS $i$-band where stars still contribute a significant fraction of the total flux while, not being negligible like in the mid-IR, the extinction corrections are still much lower than in the bluer bands.

After fixing the photometric zero points we fitted remaining two free parameters (we fixed the dust temperature to $T_{\text {dust }}=$ $250 \mathrm{~K} \mathrm{ab}$ initio): the slope $\left(\alpha_{\mathrm{UV}}\right)$ and the extinction $A_{V}^{\mathrm{PL}}$ of the obscured power-law component, and we computed the stellar extinction $A_{V}^{*}$ by forcing the FUV flux to match the observed values (even for a small amount of extinction the contribution of the obscured power-law is negligible in the FUV). We then recomputed the zero points on the extinction-corrected fluxes and iterated until the zero point of the stellar component converged to better than $1 \%$, which takes only a few iterations.

Following our work in Paper I, we experimented with two extreme extinction laws: the canonical Galactic law of Cardelli et al. (1989) - CCM, and the UV-strong SMC/30 Dor law of Gordon et al. (2003), which we refer to as GD1 following the nomenclature of STARLIGHT. Both laws are reasonably similar in the optical and near-UV, but differ significantly in the FUV. Also, while CCM shows the strong $2200 \AA$ bump characteristic of the Galaxy, the bump is absent in GD1. We verified that GD1 provides a much better fit to our PSQ data although, within the redshift range of our sample, the $2200 \AA$ bump does not affect the observations. The photometric data uncorrected for extinction are shown as blue squares in Fig. 3 while the extinctioncorrected data are shown in red, for the best fit mean stellar extinction of $A_{V}^{*}=0.27$ and the GD1 reddening law.

Figure 3 shows that our simple three component model provides a remarkably good fit to the observations over more than two decades in wavelength from the FUV to the midIR. There is clearly some degeneracy in the parameters, notably between in $\alpha_{\mathrm{UV}}$ and $A_{V}^{\mathrm{PL}}$, although we find solutions only 


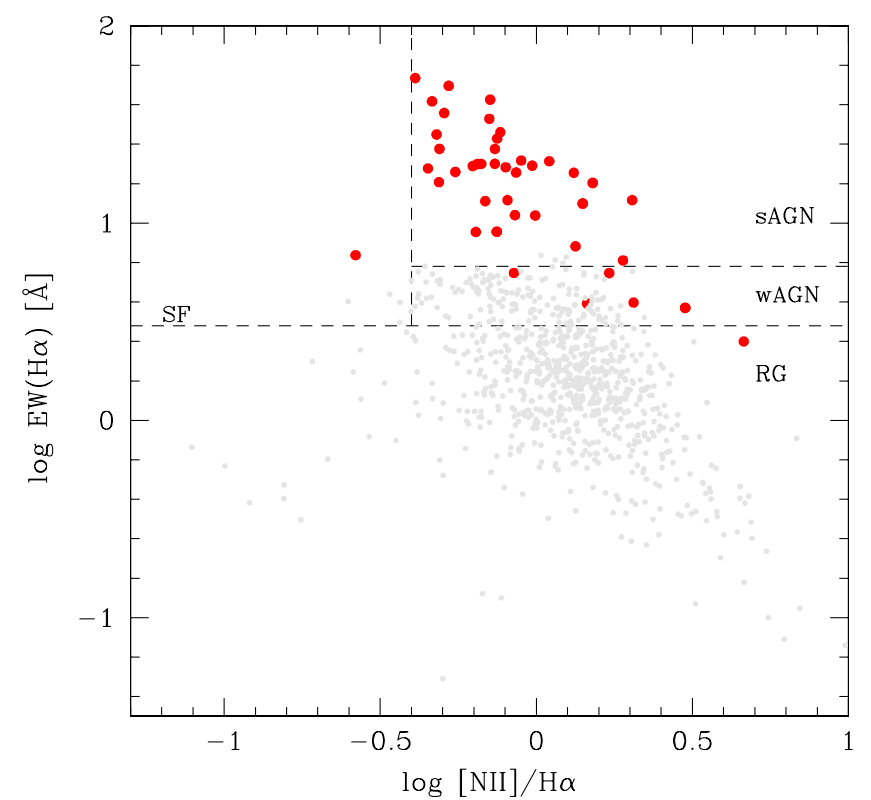

Fig. 4. WHAN diagram for the 72 PSQ in our sample. The grey points show our sample of $808 \mathrm{~K}+\mathrm{A}$ galaxies for comparison.

for $-1.2>\alpha_{\mathrm{UV}}>-1.8$ and $A_{V}^{\mathrm{PL}}>0.8$. From a visual inspection of the $\chi^{2}$ contours the best fit is obtained for $\alpha_{\mathrm{UV}}=-1.5 \pm 0.2$ and $A_{V}^{\mathrm{PL}}=1.3 \pm 0.3$. Interestingly, the power-law slope is constrained to values remarkably similar to the average value observed for X-ray luminous AGN $\left(f_{\lambda} \propto \lambda^{-1.6}\right.$; Wu et al. 2012).

This reddened power-law component is the one we included in the STARLIGHT population synthesis models described in Sect. 3.1.

\section{Emission-lines}

\subsection{Gas excitation}

Figure 4 shows the WHAN diagram described by Cid Fernandes et al. $(2010,2011)$ as a powerful emission-line diagnostic method for objects with weak emission-lines, or systems, such as ours, where the relevant Balmer lines are severely affected by underlying stellar absorption making diagnostics such as $[\mathrm{OIII}] / \mathrm{H} \beta$ (or $[\mathrm{OII}] /[\mathrm{OIII}]$ that requires extinction corrections) rather uncertain. Since most of the objects in our sample have broad $\mathrm{H} \alpha$ emission lines, we used the package PAN in IDL (Dimeo 2005) to simultaneously fit the narrow (H $\alpha$ and [NII]) and the broad components. This was only possible for a subset of all our objects because at their redshifts the SDSS spectra around $\mathrm{H} \alpha$ are rather noisy due to contamination from atmospheric features, or in some cases outside the spectral range.

As expected (the objects were selected from a QSO catalogue) most of the objects in our sample for which we could measure the parameters fall in the region of strong (and presumably real) AGN (sAGN). A few are weak AGN (wAGN formerly LINERS), while only one PSQ appears to be powered by young stars (SF).

\subsection{Gas kinematics}

In the process of separating the broad and narrow components of $\mathrm{H} \alpha$ we found that for the majority of our objects the broad component appeared to be systematically shifted relative to the narrow components, mostly, but not in all cases, to the blue. This is illustrated in the left panel of Fig. 5 that plots the radial velocity difference between the centroids of the broad and narrow lines measured from the multiple Gaussian fits.

The right-panel of the figure shows the velocity shift between the narrow components (in most cases measured from the two [OIII]4959, 5007 lines) and the stellar lines as measured by STARLIGHT from cross-correlation with the best fitting stellar templates. Negative values correspond to gas moving towards the observer and positive values to gas moving away from the observer relative to the stars. From repeated fits and from the two [OIII] emission lines we estimate the (star-gas) radial velocity differences to be accurate to $\pm 20 \mathrm{~km} \mathrm{~s}^{-1}$, so the figure shows a clear excess of blue shifted gas emission.

In general the $\mathrm{H} \beta$ emission line in PSQ is either completely absorbed by the underlying absorption, or broad and rather weak, but for some cases $\mathrm{H} \beta$ is strong and narrow showing clear P Cygni profiles. Some of these cases are shown Fig. 6 that show blue-shifted emission relative to the stellar line, confirming the predominance of blue shifts for the emission lines.

\subsection{Black-hole masses and X-ray luminosity}

The simplest way to determine black-hole (BH) masses for our PSQs is either from the Eddington luminosities, or from the masses of the spheroidal component of the host galaxies. The statistical study of X-ray luminous AGN by Wu et al. (2012) provides a useful way to estimate the Eddington ratio $\eta_{\text {Edd }}=$ $L_{\mathrm{Bol}} / L_{\mathrm{Edd}}$ from the slope of the power-law continuum in the UV. The average power-law slope for our sample $\alpha_{\mathrm{UV}}=-1.5$ (in $f_{\lambda}$ units) is typical for the QSOs in the sample of Wu et al. (2012) and indicates an Eddington ratio $\eta_{\text {Edd }} \sim 0.3$.

Runnoe et al. (2012) provide bolometric luminosity corrections for quasars at $0.145 \mu$ and $0.3 \mu$. Assuming a power-law slope $\alpha_{\mathrm{UV}}=-1.5$ for all the objects in our sample, we can use the dereddened power-law luminosities at these wavelengths to estimate the bolometric luminosities using the recommended relations of Runnoe et al. (2012). We then use the Eddington luminosity, $L_{\mathrm{Edd}}=3 \times 10^{4} M_{\mathrm{BH}}$ in solar units, to compute $\mathrm{BH}$ masses $M_{\mathrm{BH}}^{\mathrm{Edd}}$ as

$$
M_{\mathrm{BH}}^{\mathrm{Edd}}=\frac{L_{\mathrm{Bol}}}{3 \times 10^{4} \eta_{\mathrm{Edd}}} M_{\odot},
$$

where as before $\eta_{\text {Edd }}$ is the Eddington ratio. The resulting distribution of $\mathrm{BH}$ masses is shown by the black histogram in Fig. 7.

The second histogram in red shows the $\mathrm{BH}$ masses estimated from the mass of the spheroidal component $M_{\text {sph }}$ using the total stellar masses $M_{*}$ from the population synthesis models as a proxy for $M_{\mathrm{sph}}$. For simplicity we assumed $M_{\mathrm{sph}}=0.85 M_{*}$ for all our objects, and used the correlation between $M_{\mathrm{sph}}$ and $M_{\mathrm{BH}}$ from Scott et al. (2013): $\log M_{\mathrm{BH}}^{*}=0.97 \log \left(M_{\mathrm{sph}} / 3 \times 10^{11}\right)+$ 9.27 to infer $\mathrm{BH}$ masses.

Two distributions in Fig. 7 are in remarkably good agreement considering the approximations that we have made, in particular using the same power-law and the same stellar mass to bulge mass ratios for all our objects. Still, the Eddington ratio $\eta_{\text {Edd }}=$ 0.3 depends only weakly on the UV power-law slope (Wu et al. 2012), while if anything the assumption of $M_{\mathrm{sph}}=0.85 M_{*}$ is rather conservative.

The largest uncertainty arises because a substantial fraction of the total luminosity at $0.55 \mu$ is contributed by the powerlaw component (Fig. 3). So, in order to calculate the total stellar masses of each object, we have assumed that for all the objects in our sample the power-law component contributes $55 \%$ of the 

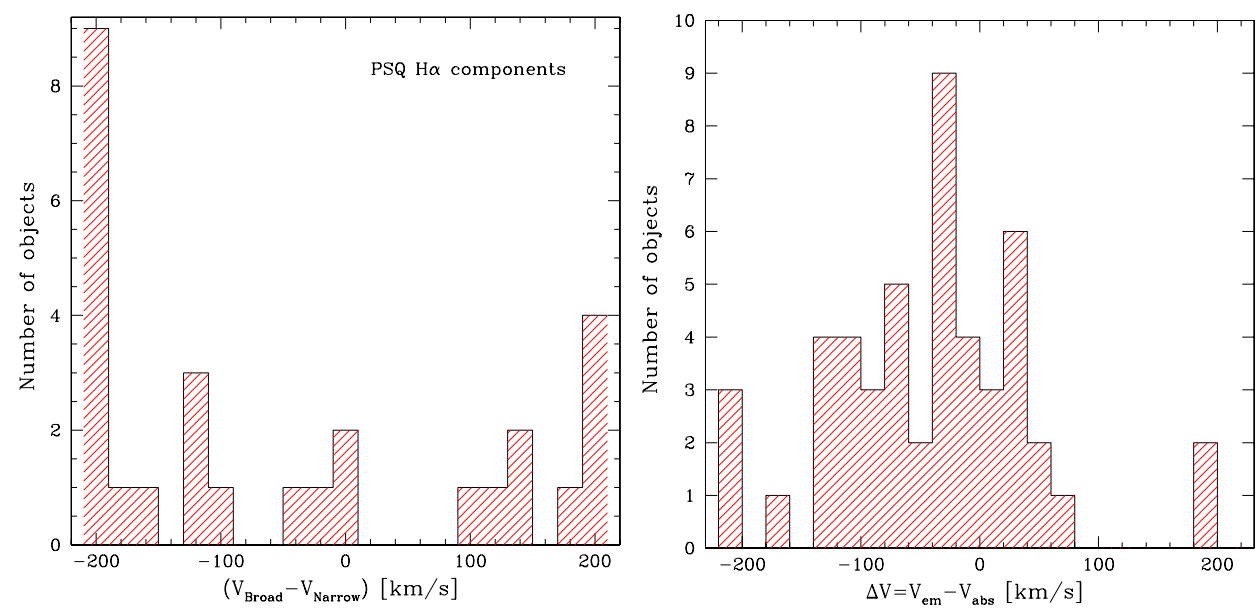

Fig. 5. Left: radial velocity differences between the broad and narrow components of the $\mathrm{H} \alpha$ line measured fitting multiple Gaussians. Right: histogram of the difference in radial velocity between the emission lines $\left(V_{\mathrm{em}}\right)$ and the absorption lines $\left(V_{\text {abs }}\right)$. The measurement errors are about $\pm 20 \mathrm{~km} \mathrm{~s}^{-1}$.
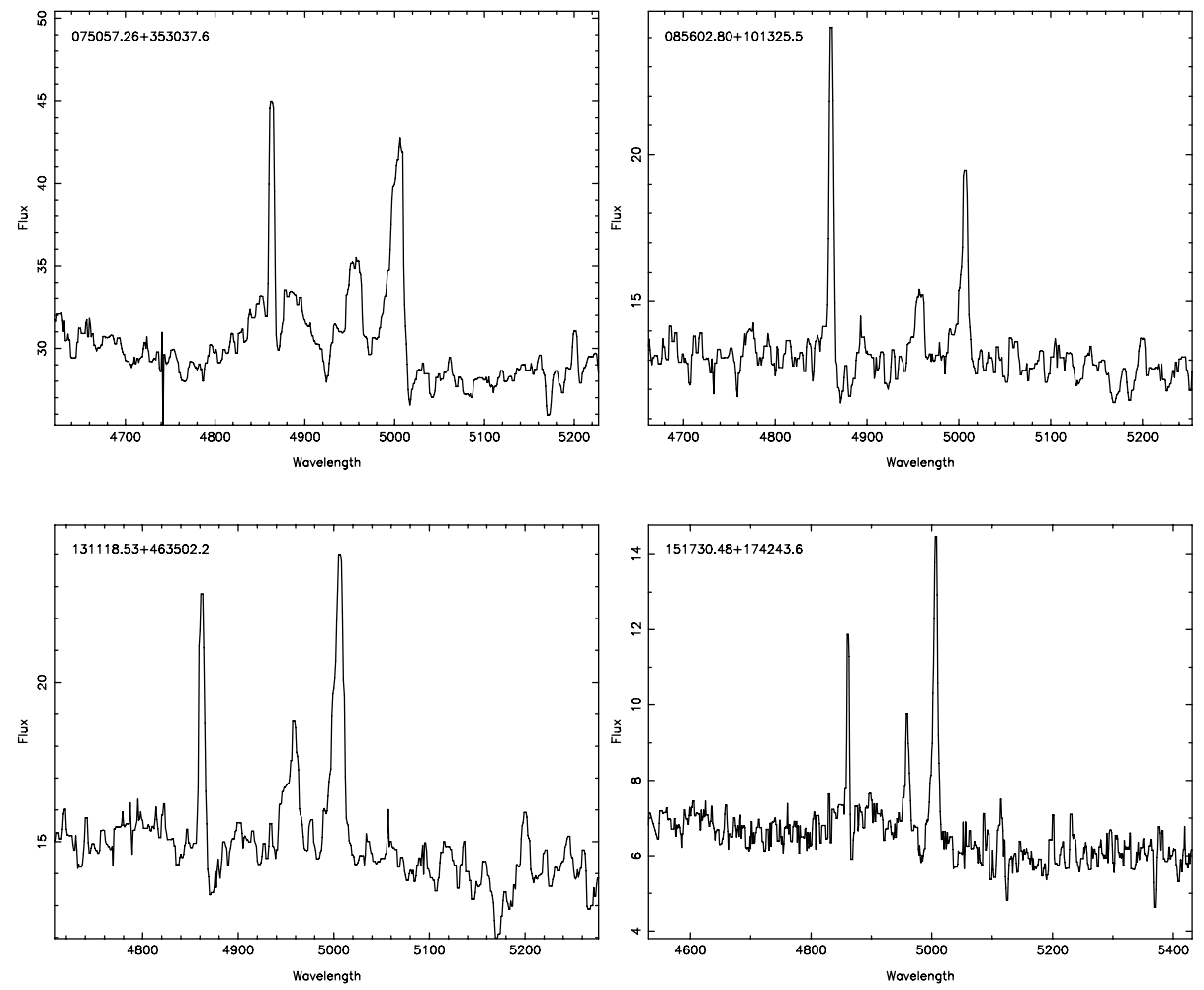

Fig. 6. Examples of some of the few objects in our sample that have narrow and relatively strong $\mathrm{H} \beta$ emission lines. The P Cygni profiles show that the gas is blue shifted relative to the stellar component.

visible light. This corresponds to the ratio of stellar to powerlaw luminosity of $L_{*} / L_{\mathrm{PL}}=0.81$ observed for the mean of our sample (Fig. 3).

Cales et al. (2013) studied a sample of 38 PSQ with substantially better $\mathrm{S} / \mathrm{N}$ spectra, and using the more standard method of combining line widths and optical luminosities. Their masses for 12 objects in common are comparable, within the uncertainties, with our $M_{\mathrm{BH}}^{*}$ values, indicating that the assumptions that went into our calculations are not entirely unreasonable.

An independent test is to use the X-ray luminosities derived from the bolometric corrections. Runnoe et al. (2012) give two separate relations for radio-loud and radio-quiet objects. All the PSQ in our sample are in the NVSS (Condon et al. 1998) and, as expected, the vast majority are radio quiet. Only six objects in our sample are radio loud with fluxes well in excess of $3 \mathrm{mJy} /$ beam, which seems to be consistent with the fact that at similar luminosities only about $10 \%$ of all QSOs are radio loud (Padovani 1993). Inverting the bolometric correction for radioquiet QSOs: $\log L_{\mathrm{Bol}}=33.08+0.29 \log L_{2-10 \mathrm{keV}}$ we get the distribution of 2-10 keV X-ray luminosities shown in Fig. 8.
At these luminosities we would expect only three of our objects to be detected by ROSAT, while none appears in the ROSAT all sky catalogue (Voges et al. 1999), which at the mean redshift of our sample has a $3 \sigma$ detection limit of $\log L_{2-10 \mathrm{keV}} \sim 44.3 \mathrm{erg} / \mathrm{s}$. Nine of our PSQ are in the XMM or Chandra archives, but only two (J025938.15+004206.4 and $\mathrm{J} 074524.97+375436.6)$ are robust detections, and a third $(\mathrm{J} 094820.38+582526.4)$ is an upper limit. The mean X-ray luminosity of these objects, $\log L_{X}=43.2 \mathrm{erg} / \mathrm{s}$, is consistent on average with our estimates from the bolometric luminosities ( $\left.\log L_{X}=43.1\right)$. We can confidently conclude, therefore, that the AGN in PSQ appear to be normal from the point of view of their $\mathrm{BH}$ masses, and their radio and X-ray luminosities.

\section{Discussion}

\subsection{Kinematics}

In the scenario of Hopkins et al. (2008a), the QSO phase is preceded by the Antennae/ULIRG phases characterised by the formation of numerous starburst clusters and super-clusters in the 


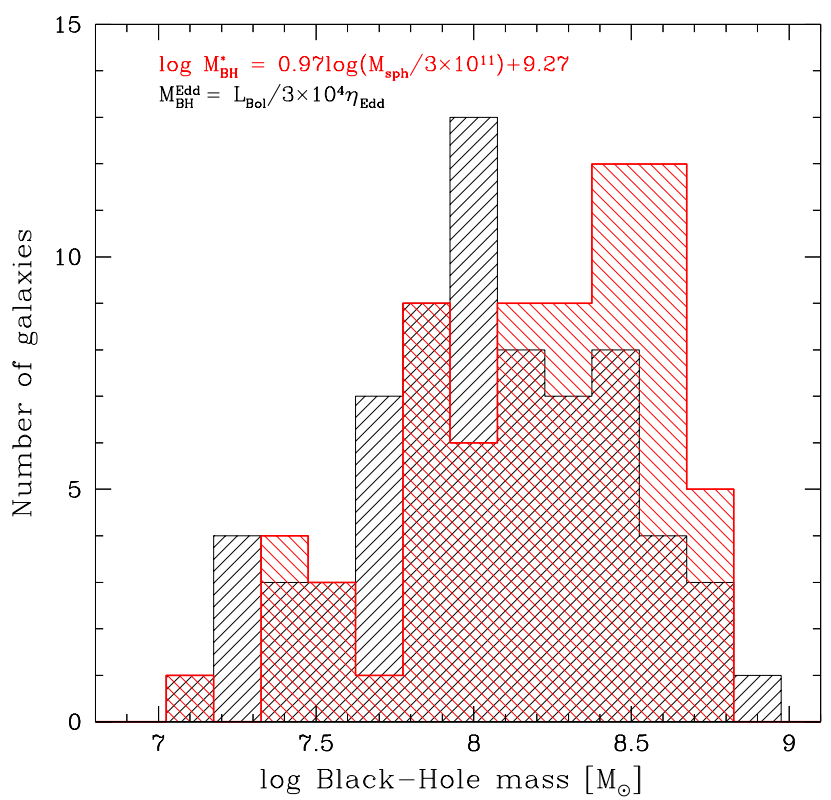

Fig. 7. Left: black-hole masses calculated with two different methods shown in the lagend. The histogram in black (left) was calculated using the Eddington ratios and the bolometric luminosities. The histogram in red (right) shows the distribution of masses calculated using the relation between $M_{\mathrm{BH}}$ and $M_{\mathrm{sph}}$. Right: correlation between $M_{\mathrm{BH}}^{\mathrm{Edd}}$ and $M_{\mathrm{BH}}^{*}$. The solid line shows a least-squares fit with coefficients indicated in the legend.

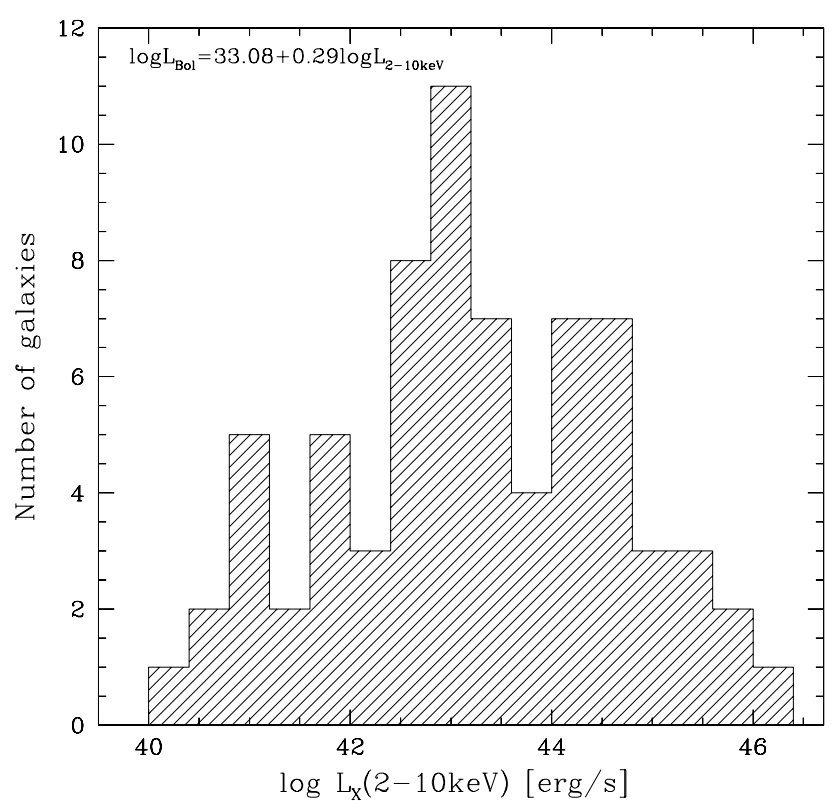

Fig. 8. Distribution of X-ray luminosities for PSQ calculated using the bolometric corrections for radio-quiet QSOs shown in the legend.

arms of both spiral components. While large amounts of molecular material may be channelled to the nuclear regions by tidal forces during the merger, nuclear starbursts in these phases are either still weak, or heavily enshrouded in dust, such that their emission is not visible at optical and near-IR wavelengths. By the time massive starburst activity is finally ignited in the nucleus, all star formation in the arms would have ceased leaving behind a large intermediate age population of stars distributed across the remains of the tidally distorted spiral arms.
Therefore, while the emission lines in PSQ mostly arise from the nuclear regions (e.g. Sanmartim et al. 2014), the stellar lines originate mostly from the remnants of massive starburst clusters in the spiral arms, so it should come as no surprise to observe a wide distribution of radial velocity shifts between stars and gas. And, since these shifts depend mostly on the - in principle random - orientation of the merger with respect to the line of sight, we would expect to observe a uniform distribution of velocityshifts centred around zero. However, as shown in the right panel of Fig. 5, while we do observe a uniform distribution, the gas appears to be systematically blue shifted relative to the stars.

On the other hand, we observe a rather messy distribution of relative velocities between the narrow and broad components of $\mathrm{H} \alpha$, with the broad components either blue-shifted or redshifted relative to the narrow lines. Therefore, if the broad-line emitting region (BLR) is at rest relative to the systemic velocity of the galaxy, the centroid shifts we observe must be due to a combination of extinction and inflow, outflow, or rotation of the BLR.

For a spherical geometry, the predominance of blue shifts in our sample would indicate a net outflow of the BLR, contrary to the model of Gaskell \& Goosmann (2013), but other geometries, such as an inclined rotating disk plus an oblique obscuring torus, could also provide the variety of blue and red shifts observed in PSQ (Stern et al. 2015). Beyond asserting that there are ways to explain the observations of PSQ, however, the kinematics of the BLR is a very complex issue that is well beyond the scope of this paper.

Nevertheless, we may safely conclude that the distribution of the relative velocities between the narrow emission lines and the stars result from a combination of rotation plus a net outflow (in most cases) of the narrow emission line emitting gas. This conclusion agrees with the spatially resolved observations of the nearby PSQ J0330-0532 by Sanmartim et al. (2014), although this object is not in our catalogue as it hosts a rather low population of intermediate-age stars.

\subsection{Nuclear star formation}

We have seen that the intermediate-age stellar populations observed in $\mathrm{K}+\mathrm{A}$ galaxies are not in place in PSQ, where intermediate-age stars are both less abundant and significantly metal poorer. We are forced to conclude, therefore, that either PSQ are not the immediate progenitors of $\mathrm{K}+\mathrm{A}$ galaxies, or that the bulk of the young stars in PSQ are still being formed. This requires that vast amounts of metal-enriched molecular gas be funnelled onto the central regions during the merger in order to fuel a huge nuclear starburst, which is still not visible in PSQ. (Unfortunately, the SDSS spectra of our objects lack the S/N and resolution required to infer the metallicities of the nebular gas in PSQ and discern nuclear stellar features, but we do know that in $\mathrm{K}+$ As the little nebular gas that remains has well over solar metallicities.)

The star formation rates of these putative nuclear starbursts would need to reach $10-100$ solar masses per year $\left(\sim 10^{10} M_{\odot}\right.$ in less than a few $100 \mathrm{Myr}$ ) and be extremely compact, perhaps extending over not much more than a few kpc (Sanmartim et al. 2014). Under these conditions it would seem reasonable to expect that much of the luminosity that we observe as an obscured power-law component in PSQ would be produced not by an AGN, but by a very massive nuclear starburst. At an age of a few Myr the bolometric mass-to-light ratio of a simple starburst is about $M / L_{\mathrm{Bol}} \sim 3 \times 10^{-4}$ (Leitherer 1999), so the mean bolometric luminosity of the power-law component in our PSQ, 
$L_{\mathrm{Bol}}=10^{44.9} \mathrm{erg} \mathrm{s}^{-1}$, would imply a total mass of about $10^{8} M_{\odot}$ in young stars, about two orders of magnitude lower than the "missing" intermediate-age stellar population in PSQ.

An additional challenge for this scenario is that the young stars must form over time scales much shorter than $10 \mathrm{Myr}$, which requires a very compact concentration of molecular gas. Compact nuclear starburst clusters may resemble type-I AGN as a consequence of supernovae evolving in a high density interstellar medium (Terlevich et al. 1992), so a critical test of this Starburst-AGN connection is that at least some PSQ should have huge concentrations of cold, metal-rich molecular gas in their nuclear regions making them extremely luminous $\mathrm{CO}$ sources.

\subsection{Comparison with previous investigations}

Canalizo \& Stockton (2001) have studied a sample of transitional AGN which seem to be objects in transition between the (U)LIRG and the PSQ phase. In Canalizo \& Stockton (2013) they extended their study to more normal QSOs and consistently find that the intermediate-age populations of their objects - that they associate with remnants of major mergers - are offset from the nuclei by typically $\sim 8 \mathrm{kpc}$. This is consistent with our conjecture that the intermediate-age populations in PSQ must be the remnants of starburst clusters in the arms of the merging galaxies.

Our results are in broad agreement with the study of Cales et al. (2013) who observed 38 PSQ selected from the SDSS, in particular with our conclusion that the putative AGN in PSQ coexist with massive bursts of star formation. Unfortunately, there are only 12 objects in common between our two samples. Ten of the objects in the Cales et al. (2013) sample are not in the DR7Q, while their selection criterion $E W(\mathrm{H} \delta)>1 \AA$ is substantially less stringent than our criterion of $E W(\mathrm{H} \delta)>3 \AA$, so it admits objects that we would not classify as PSQ.

While as expected the majority of our objects fall in the strong AGN (sAGN) domain of the WHAN diagnostic diagram, most of the objects in the Cales et al. sample appear to be either LINERS or star-forming, with the caveat that they use the $[\mathrm{OIII}] / \mathrm{H} \beta$ vs. $[\mathrm{NII}] / \mathrm{H} \alpha$ BPT plane to classify their objects. In PSQ $\mathrm{H} \beta$ is rather weak and extremely sensitive to corrections for underlying absorption, so using BPT diagrams involving $\mathrm{H} \beta$ seems risky. However, the $\mathrm{BH}$ masses derived by Cales et al. (2013) using a completely different methodology are substantially similar to the $\mathrm{BH}$ masses of our PSQ, and this indicates that, even thought the stellar populations may differ, the AGNs themselves seem to be similar.

In their study of J0330-0532, Sanmartim et al. (2014) found that the intermediate-age population avoids the nuclear regions and is distributed along a disk-like structure, which would be consistent with our results, although as mentioned above, this object does not meet our selection criterion for PSQ.

\section{Conclusions}

We have compiled a sample of 72 bona fide post-starburst QSOs (PSQ) selected from the SDSS DR7 quasar catalogue purely on the basis of the equivalent width of the Balmer $\mathrm{H} \delta$ lines. We have used published photometry and SDSS spectroscopy to study the stellar populations, gas properties, and SEDs of the sample. Our main conclusion is that PSQ do not (yet?) contain the large population of metal-rich intermediate-age stars that characterise poststarburst $(\mathrm{K}+\mathrm{A})$ galaxies. Thus, either PSQ are not the immediate progenitors of $\mathrm{K}+\mathrm{As}$, or they contain hugely massive nuclear starbursts where the bulk of their intermediate-age stars are still being formed.

Our results, however, are based on rather low S/N SDSS spectra of these relatively faint and distant objects. Further progress in the understanding of PSQ, therefore, requires substantially deeper observations in order to refine the population synthesis models and to investigate the kinematics and physical conditions of the nebular gas as well as to peek into the nuclear regions in search for the hidden AGN.

The majority of our PSQ are not strong radio or X-ray sources, although the number of objects observed in X-rays remains rather small. Thus, deep X-ray and mm wave observations are critically needed to establish the nature of the AGN in these objects and to check for the presence of nuclear starbursts. The present paper reports the initial steps of such research programme.

Acknowledgements. J.M. acknowledges the award of Special Visiting Researcher fellowship of the Ciencia sem fronteiras programme of the Brazilian government through their federal funding agencies - CNPq. J.M. also thanks the hospitality of Nanjing University where the initial steps of this research programme were outlined. We are grateful to Roberto Cid-Fernandes the father of STARLIGHT for continuous support in the use of the code. The STARLIGHT project is supported by the Brazilian agencies CNPq, CAPES and FAPES and by the France-Brazil CAPES/Cofecub programme. We thank our anonymous referee for very useful comments that led to a much improved version of the paper. This research has made use of the NASA/ IPAC Infrared Science Archive, which is operated by the Jet Propulsion Laboratory, California Institute of Technology, under contract with the National Aeronautics and Space Administration. The entire GALEX Team gratefully acknowledges NASA's support for construction, operation, and science analysis for the GALEX mission, developed in corporation with the Centre National d'Études Spatiales of France and the Korean Ministry of Science and Technology. We acknowledge the dedicated team of engineers, technicians, and administrative staff from JPL/Caltech, Orbital Sciences Corporation, University of California, Berkeley, Laboratoire d'Astrophysique Marseille, and the other institutions who made this mission possible. Funding for the SDSS and SDSS-II has been provided by the Alfred P. Sloan Foundation, the Participating Institutions, the National Science Foundation, the U.S. Department of Energy, the National Aeronautics and Space Administration, the Japanese Monbukagakusho, the Max Planck Society, and the Higher Education Funding Council for England. The SDSS Web Site is http://www.sdss.org/. The SDSS is managed by the Astrophysical Research Consortium for the Participating Institutions. The Participating Institutions are the American Museum of Natural History, Astrophysical Institute Potsdam, University of Basel, University of Cambridge, Case Western Reserve University, University of Chicago, Drexel University, Fermilab, the Institute for Advanced Study, the Japan Participation Group, Johns Hopkins University, the Joint Institute for Nuclear Astrophysics, the Kavli Institute for Particle Astrophysics and Cosmology, the Korean Scientist Group, the Chinese Academy of Sciences (LAMOST), Los Alamos National Laboratory, the Max-Planck-Institute for Astronomy (MPIA), the Max-Planck-Institute for Astrophysics (MPA), New Mexico State University, Ohio State University, University of Pittsburgh, University of Portsmouth, Princeton University, the United States Naval Observatory, and the University of Washington. This publication makes use of data products from the Two Micron All Sky Survey, which is a joint project of the University of Massachusetts and the Infrared Processing and Analysis Center/California Institute of Technology, funded by the National Aeronautics and Space Administration and the National Science Foundation. This publication makes use of data products from the Wide-field Infrared Survey Explorer, which is a joint project of the University of California, Los Angeles, and the Jet Propulsion Laboratory/California Institute of Technology, funded by the National Aeronautics and Space Administration. This research has made use of the NASA/IPAC Extragalactic Database (NED) which is operated by the Jet Propulsion Laboratory, California Institute of Technology, under contract with the National Aeronautics and Space Administration.

\section{References}

Abazajian, K. N., Adelman-McCarthy, J. K., Agüeros, M. A., et al. 2009, ApJS, 182,543

Bruzual, G., \& Charlot, S. 2003, MNRAS, 344, 1000 (BC03)

Cales, S. L., Brotherton, M. S., Shang, Z., et al. 2013, ApJ, 762, 90

Canalizo, G., \& Stockton, A. 2001, ApJ, 555, 719

Canalizo, G., \& Stockton, A. 2013, ApJ, 772, 132 
Cardelli, J. A., Clayton, G. C., \& Mathys, J. S. 1989, ApJ, 345, 245 (CCM)

Cid Fernandes, R., Mateus, A., Sodré, L., Stasińska, G., \& Gomes, J. M. 2005 , MNRAS, 358, 363

Cid Fernandes, R., Stasińska, G., Schlickmann, M. S., et al. 2010, MNRAS, 403, 1036

Cid Fernandes, R., Stasińska, G., Mateus, A., \& Vale Asari, N. 2011, MNRAS, 413,1687

Cisternas, M., Jahnke, K., Inskip, K. J., et al. 2011, ApJ, 726, 57

Condon, J. J., Cotton, W. D., Greisen, E. W., et al. 1998, AJ, 115, 1693

De Propris, R., Baldry, I. K., Bland-Hawthorn, J., et al. 2014, MNRAS, 444, 2200

De Propris, R., \& Melnick, J. 2014, MNRAS, 439, 2837

Dimeo, R. 2005, PAN User Guide, ftp://ftp.ncnr.nist.gov/pub/staff/ dimeo/pandoc.pdf

Gaskell, M. C., \& Goosmann, R. W. 2013, ApJ, 769, 30

Goto, T. 2006, MNRAS, 369, 1765

Gordon, K. D., Clayton, G. C., Misselt, K. A., Landolt, A. U., \& Wolff, M. J. 2003, ApJ, 594, 279 (GD1)

Hopkins, P. F., Hernquist, L., Cox, T. J., \& Keres, D. 2008a, ApJS, 175, 356

Hopkins, P. F., Cox, T. J., Keres, D., \& Hernquist, L. 2008b, ApJS, 175, 390

Jahnke, K., \& Maccio, A. V. 2011, ApJ, 734, A92

Kocevski, D. D., Faber, S. M., Mozena, M., et al. 2012, ApJ, 744, 148

Koss, M., Mushotzky, R., Veilleux, S., et al. 2011, ApJ, 739, A57

Leitherer, K., Schaerer, D., Goldader, J. D., et al. 1999, ApJS, 123, 3

Melnick, J., \& De Propris, R. 2013, MNRAS, 431, 2034

Melnick, J., \& De Propris, R. 2014, A\&A, 572, A5
Mihos, C. J., \& Hernquist, L. 1996, ApJ, 464, 641

Morrissey, P., Conrow, T., Barlow, T. A., et al. 2007, ApJS, 173, 682

Padovani, P. 1993, MNRAS, 263, 461

Runnoe, J. C., Brotherton, M. S., \& Shang, Z. 2012, MNRAS, 422, 478

Sanmartim, D., Storchi-Bergmann, T., \& Brotherton, M. S. 2014, MNRAS, 443, 584

Schneider, D. P., Richards, G. T., Hall, P. B., et al. 2010, AJ, 139, 2360 (DR7Q)

Scott, C., \& Kaviraj, S. 2014, MNRAS, 437, 2137

Scott, N., Graham, A.W., \& Schombert, J. 2013, ApJ, 768, 76

Skrutskie, M. F., Cutri, R. M., Stiening, R., et al. 2006, AJ, 131, 1163

Snyder, G. F., Cox, T. J., Hayward, C. C., Hernquist, L., \& Jonsson, P. 2011 , ApJ, 741, 77

Stern, J., Hennawi, J. F., \& Pott, J-U. 2015, ApJ, 804, 57

Teng, S. H., Schawinski, K., Urry, C. M., et al. 2012, ApJ, 753, 165

Terlevich, R., Tenorio-Tagle, G., Franco, J., \& Melnick, J. 1992, MNRAS, 255, 713

Toomre, A., \& Toomre, J. 1972, ApJ, 178, 623

Toomre, A. 1977, in Evolution of Galaxies and Stellar Populations, Proc. a Conference at Yale University, May 19-21, eds. M. T. Beatrice, \& B. L. Richard (New Haven: Yale University Observatory), 401

Urrutia, T., Lacy, M. H., \& Becker, R. H. 2008, ApJ, 674, 80

Villforth, C., Hamann, F., Rosario, D. J., et al. 2014, MNRAS, 439, 3342

Voges, W., Aschenbach, B., Boller, T., et al. 1999, A\&A, 349, 389

Wong, O. I., Schawinski, K., Kaviraj, S., et al. 2012, MNRAS, 420, 1684

Wright, N. J., Barlow, M. J., Greimel, R., et al. 2009, MNRAS, 400, 1413

Wu, J., Vanden Berk, D., Grupe, D., et al. 2012, ApJS, 201, 10 